\title{
Fabrication and Optical Properties of Silicon Nanowires Arrays by Electroless Ag-catalyzed Etching
}

Jie Tang, Jianwei Shi, Lili Zhou, Zhongquan Ma*

(Received 15 July 2011; accepted 20 July 2011; published online 13 August 2011.)

\begin{abstract}
In order to realize ultralow surface reflectance and broadband antireflection effects which common pyramidal textures and antireflection coatings can't achieve in photovoltaic industry, we used low-cost and easy-made Ag-catalyzed etching techniques to synthesize silicon nanowires (SiNWs) arrays on the substrate of single-crystalline silicon. The dense vertically-aligned Si NWs arrays are fabricated by local oxidation and selective dissolution of Si in etching solution containing Ag catalyst. The Si NWs arrays with $3 \mu \mathrm{m}$ in depth make reflectance reduce to less than $3 \%$ in the range of 400 to $1000 \mathrm{~nm}$ while reflectance gradually reached the optimum value with the increasing of etching time. The antireflection of Si NWs arrays are based on indexgraded mechanism: Si NWs arrays on a subwavelength scale strongly scatter incident light and have graded refractive index that enhance the incidence of light in usable wavelength range. However, surface recombination of Si NWs arrays are deteriorated due to numerous dangling bonds and residual Ag particles.
\end{abstract}

Keywords: Si nanowires; Ag-catalyzed etching; Broadband antireflection; Surface recombination

Citation: Jie Tang, Jianwei Shi, Lili Zhou and Zhongquan Ma, "Fabrication and optical properties of silicon nanowires arrays by electroless Ag-catalyzed etching", Nano-Micro Lett. 3 (2), 129-134 (2011). http://dx. doi.org/10.3786/nml.v3i2.p129-134

\section{Introduction}

As a promising candidate for conventional fossil energy, high-efficiency and low-cost silicon photovoltaic devices still meet some challenges such as non-abundant utilization of solar spectrum [1] and various carrier recombination [2] in spite of rapid development over the past few decades. In order to improve the perfomances of photovoltaic devices, novel ideas and advanced technologies should be applied to present devices. Considering of fast progress in the field of nanotechnology, low dimensional silicon nanostructures with tunable opto-electronic properties have great potential in photovoltaic conversion. For example, silicon nanowires (Si NWs) arrays have been proven to enhance optical absorption [3] and their radial pn junction where solar cells offer efficient carrier collection [4]. Compared with common pyramids and antireflection coatings on singlecrystalline silicon solar cells, density-graded porous $\mathrm{Si}$ NWs arrays with subwavelength pattern exhibit broadband antireflection effects [5]. Similar to other subwavelength structures (SWS) such as moth eye [6] and surface-relief grating [7], Si NWs arrays have gradient refractive index (RI) and its antireflection is based on optical-constant gradient mechanism [8]. As most practical photovoltaic modules use c-Si materials with limited spectrum, optimized reflectance of Si NWs arrays help promote their output power. Up to now, main fabrication techniques of Si NWs arrays include bottom up approach by solution processing, physical or chemical vapour deposition and top down approach by dry or wet etching [9]. In particular, electroless Agcatalyzed etching (EAE) which uses HF solution containing Ag particles to selectively etch silicon surface 
[10] is not only able to be used for large-scale wafers but also cheap and easy-made. Since then, Si NWs arrays by EAE technique are suitable for widespread application to photovoltaic industry and some other fields such as electronic biosensors [11], light-emitting diodes [12], field-effect transistors [13] and so on.

Herei, we reported an Ag-catalyzed etching method which was used to synthesize Si NWs arrays on singlecrystalline Si substrates and analyzed the evolution and fabrication of Si NWs arrays during the etching procedure. The broadband antireflection characteristics of Si NWs arrays were measured to determine the optimized reflectance and their antireflection mechanism was explained by analyzing the relationships between morphologies and reflection of Si NWs arrays. Furthermore, the influence of Ag-catalyzed etching on the surface recombination was given and possible factors facilitating recombination were discussed.

\section{Experimental details}

The typical fabrication of Si NWs arrays on Si substrates includes four steps: I) the commercial p-type (100) CZ-Si wafers with resistivity of $\sim 1.5 \Omega \cdot \mathrm{cm}$ and thickness of $200 \mu \mathrm{m}$ were cut into $2 \times 2 \mathrm{~cm}^{2}$ squares, and then they were dipped into $1: 8 \mathrm{HF} / \mathrm{HNO}_{3}$ solution for 2 minutes at room temperature to remove surface sawdamage and rinsed with deionized water thoroughly; II) Ag-particles catalyst layers were plated on the cleaned surfaces by immersing the $\mathrm{Si}$ substrates into the mixture of 4.6 $\mathrm{M} \mathrm{HF}$ and $0.01 \mathrm{M} \mathrm{AgNO}_{3}$ aqueous solution at $30^{\circ}$ for 1 minutes; III) the $\mathrm{Si}$ pieces wrapped by $\mathrm{Ag}$ thin films were etched with aqueous solutions of 4.6 $\mathrm{M} \mathrm{HF}$ and $0.02 \mathrm{M} \mathrm{H}_{2} \mathrm{O}_{2}$ at $30^{\circ}$ for different etching times; iv) the samples were successively immersed into diluted $\mathrm{HNO}_{3}$ to remove residual $\mathrm{Ag}$, rinsed with deionized water and dried by blowing $\mathrm{N}_{2}$. All chemicals were analytical reagent grade and the resistivity of deionized water was $18.2 \mathrm{M} \Omega \cdot \mathrm{cm}$.

A FEI QUANTA 250 FEG field emission scanning electron microscope (SEM) and JEM 2010F transmission electron microscope (TEM) were used to observe the surface and cross-sectional morphologies of Si NWs arrays on Si wafers as well as the morphology of a single nanowire. A TU 1901 UV-VIS spectrophotometer with an integrating sphere was used to measure the reflectance of Si NWs arrays in order to determine their broadband antireflection efffect. A WT-2000 minority carrier lifetime tester with microwave photoconductivity decay technology (MW-PCD) was employed to characterize surface recombination of the samples.

\section{Results and discussion}

Figure 1 shows the SEM images of Ag-particles layers plated on the surface of Si wafers as above mentioned step II. The Ag film consists of white and bright dentrities which lie loosely upon the substrates (see Fig. 1(a)). The thickness of the film is about $1.2 \mu \mathrm{m}$ according to the cross-sectional image shown in Fig. 1(b). The highmagnified image (see Fig. 1(c)) indicates that there formed interconnected network which composed of $\mathrm{Ag}$ patches. When the etching process of $\mathrm{H}_{2} \mathrm{O}_{2}$ and $\mathrm{HF}$ in step III is skiped, the morphology after removing $\mathrm{Ag}$ residuals was shown in Fig. 1(d), where the pits and hills are randomly scattered. As discussed by Ogata [14], the plating of Ag catalysts in etching solution was a galvanic procedure in which the $\mathrm{Ag}$ ions input holes into the valence band of the Si wafer due to the difference of Fermi levels of these two substances and the reduced Ag particles formed nuclei in micro- or nanorange on the surface of the substrate. The Ag nuclei subsequently connected together and made up the dendrite-like structures on the networks. Such distribution of $\mathrm{Ag}$ catalyst leads to the appearence of uneven plane whose formation will be indicated in details later. The $\mathrm{AgNO}_{3}$ and $\mathrm{HF}$ solution in step II mainly plays a role in plating $\mathrm{Ag}$ process but the etching speed is very slow. As a result, the $\mathrm{H}_{2} \mathrm{O}_{2}$ and $\mathrm{HF}$ etching was employed after Ag catalysts were plated.

The surface and cross-sectional morphology of the sample etched as step III for 10 minutes are shown in Fig. 2(a) and (b). The textured surface is composed of dense one-dimensional nanowires that have bright contrast with substrate and are uniformly spaced by pits among them. The depth of the Si NWs arrays and diameters of pits are separately about $500 \mathrm{~nm}$ and 200$500 \mathrm{~nm}$. Almost all nanowires are vertical to substrate in the direction of (100) and few nanowires collapse because of failure to support their weight. The depth of the Si NWs arrays increases with the increasing of etching time. For example, Si NWs arrays etched for one hour have the thickness of $3 \mu \mathrm{m}$ (see Fig. 2(c)). As revealed in the TEM image of a single nanowire (Fig. 2(d)), the width of the nanowire is about $200 \mathrm{~nm}$ and one end of the nanowire are a little bigger than the other end.

Although up to now the mechanism of Ag-catalyzed etching procedure is still unproven by experiments in details, the evolution and fabrication of Si NWs arrays can be deduced according to the growth theory proposed by Peng [15].

While the $\mathrm{Ag}$ nucleis on nanoscales was plated on the surface of Si substrate in etching solution, the holes input to valence band of $\mathrm{Si}$ by previous $\mathrm{Ag}$ ions help oxidize Si surfaces underneath the Ag nuclei. The silicon oxide formed in the process of local oxidation was immediately dissolved in the solution owing to HF etching and this leaded to the appearance of pits or pores which the Ag particles entered. The schematic diagram of the whole etching process is indicated as Fig. 3 and 
the overall reaction was under way as follow: $4 \mathrm{Ag}^{+}+$ $\mathrm{Si}+6 \mathrm{~F}^{-} \rightarrow 4 \mathrm{Ag}+\mathrm{SiF}_{6}^{2-}$. Owing to the unique preferential catalyzation of the $\mathrm{Ag}$ catalyst at the bottom of the $\mathrm{Ag} / \mathrm{Si}$ interface, longitudinal etching perpendicular to the surface was much faster than the lateral etching parallel to the surface. As a result, the Ag-catalyzed etching selectively proceeded along the crystallographic orientation of the surface and the arrangemnt pattern of the Ag catalyst on the surface of Si decided on the porosity and dimension of the Si NWs arrays.

As we all know, coarse surfaces shine less than flat ones and nanostructured surfaces with high roughness often appear black which indicates low reflectance. Our samples textured with Si NWs arrays have completely black surfaces and reflectance measurements are given in Fig. 4. For the sample that was etched without HF and $\mathrm{H}_{2} \mathrm{O}_{2}$, the reflectance is generally more than $25 \%$ in the whole band and is especially high in short-
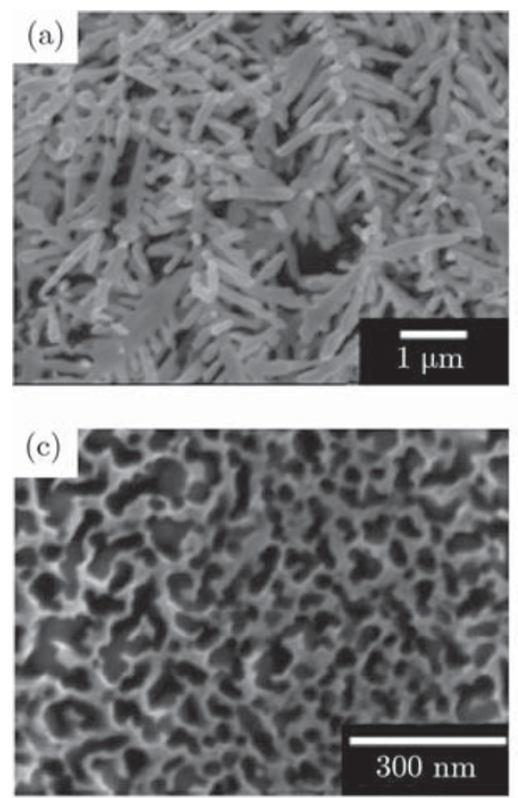
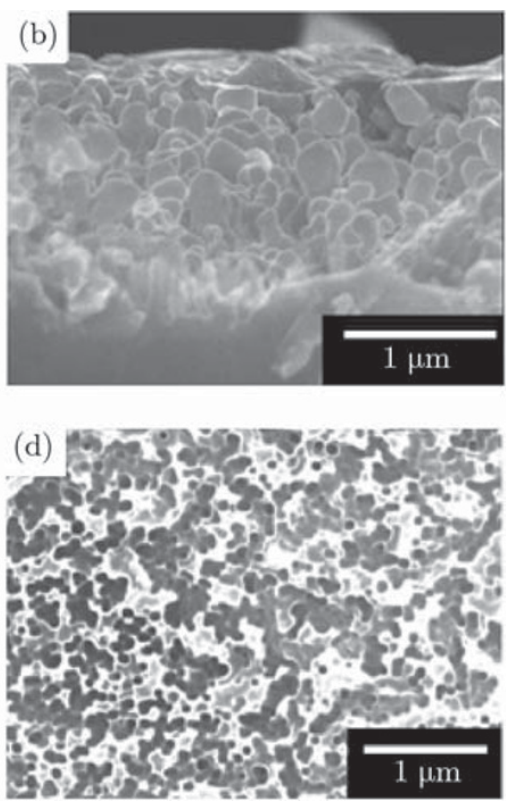

Fig. 1 (a) and (b) Surface and cross-sectional morphology photo by SEM after Ag particles were plated on the surface; (c) local high magnification picture of (a); (d) SEM picture of the surface without $\mathrm{H}_{2} \mathrm{O}_{2}$ and HF etching after $\mathrm{Ag}$ particles were removed.
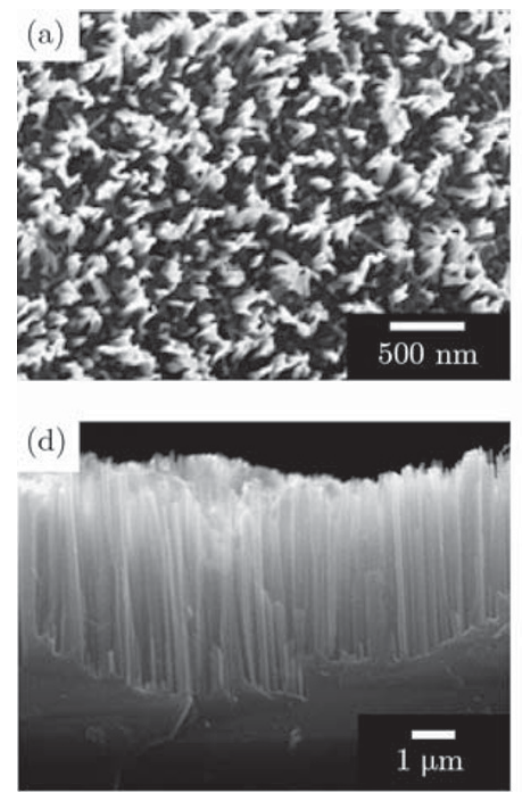
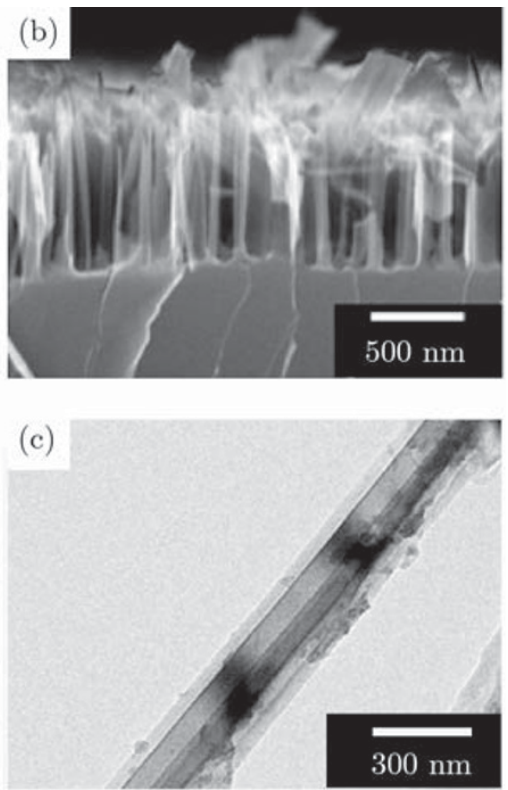

Fig. 2 (a) and (b) Surface and cross-sectional morphology photo by SEM after etched by $\mathrm{H}_{2} \mathrm{O}_{2}$ and $\mathrm{HF}$ for 10 minutes; (c) SEM picture of the cross section after etched by $\mathrm{H}_{2} \mathrm{O}_{2}$ and $\mathrm{HF}$ for one hour; (d) TEM picture of a single nanowire. 

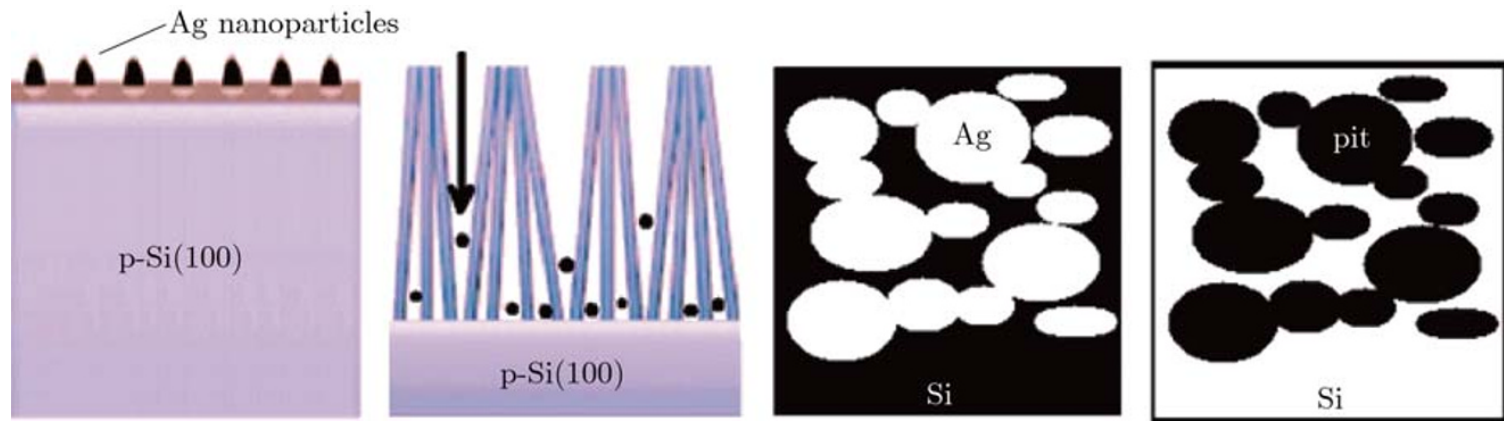

Fig. 3 Schematic diagram about evolution and fabrication of Si NWs arrays by Ag-catalyzed etching.

wavelength range. The effectless antireflection is attributed to low surface roughness since pits are shallow as shown in Fig. 1(d). In addition, the high reflectance in short-wavelength range is because refractive index of Si substrate is higher in this range than the average value.

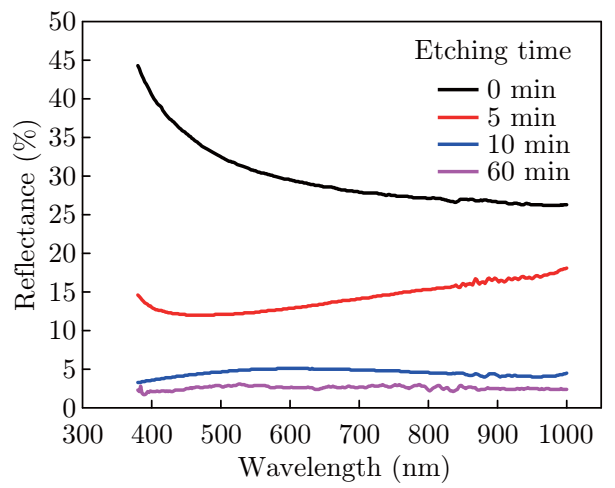

Fig. 4 Reflectance spectra of the samples etched by $\mathrm{HF}$ and $\mathrm{H}_{2} \mathrm{O}_{2}$ for different times.

For the $3 \mu \mathrm{m}$ deep Si NWs array etched for one hour by $\mathrm{HF}$ and $\mathrm{H}_{2} \mathrm{O}_{2}$, the reflectance curve tends to be plane and its values are no more than $3 \%$ from 400 $\mathrm{nm}$ to $1000 \mathrm{~nm}$. Such excellent antireflection effects are based on the interaction between incident light and the Si NWs arrays (Fig. 5). The diameters and spacing of Si NWs are smaller than the wavelength of incident visible light, so the diffuse scattering of incident light is strong. Light propagates along a curved path in the inhomogeneous mixtures of Si substrates and air on a subwavelength scale. According to effective medium theory (EMT) first proposed by Garnett [16], the Si NWs arrays can be approximated to multilayer "effective medium" (EM) and the effective refractive index (RI) of EM increases with decreasing of volume fraction of the materials. Thus, the RI of the Si NWs arrays gradually increases from 1 at the top near the air to about 3.5 at the bottom near the Si substrates. Such materials with gradient-index reduce surface reflection to an ultra-low level because an interface between any two adjacent EM with matching RI almost doesn't shine. Whether light incident on the surfaces of
Si NWs arrays is short-wavelength or long-wavelength, their RI profile is always graded. Therefore, ultralow surface reflectance and broadband antireflection effect are accomplished by index-graded $\mathrm{Si} \mathrm{NW}$ s arrays on subwavelength scale. The weighted average reflectance $\mathrm{R}_{w}$ which considers solar spectrum from $400 \mathrm{~nm}$ to 1000 $\mathrm{nm}$ is calculated to be $2.8 \%$ according to the definition $\mathrm{R}_{w}=\int \mathrm{F}(\lambda) \mathrm{R}(\lambda) \mathrm{d} \lambda$ where $\mathrm{F}(\lambda)$ is incident photon flux.

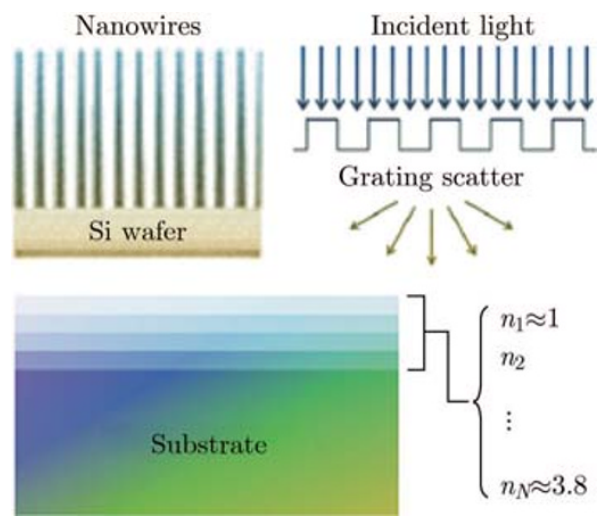

Fig. 5 Schematic diagram of broadband antireflection mechanism based on graded refractive index.

With the increasing of etching time of $\mathrm{HF}$ and $\mathrm{H}_{2} \mathrm{O}_{2}$ in step iii, the reflectance is firstly suppressed in shortwavelength range for shallow texture like the sample etched for 5 minutes and then suppressed in longwavelength range for deep texture like the sample etched for 10 minutes. In the light of the graded-density surface texture model provided by Stephens [17], the reflectance of index-graded textures on subwavelength scale depends on the ratio of $d$ (depth of textures) and $\lambda$ (incident wavelength) and the exponential relationship was given as $\mathrm{R}(\mathrm{d}, \lambda)=\mathrm{R}_{0}(\lambda) \exp [-\mathrm{C}(\mathrm{d} / \lambda)]$ where $\mathrm{R}_{0}(\lambda)$ is the reflectance of flat surface and $\mathrm{C}$ is a constant. For the texture like the sample etched for 5 minutes, its depth is much smaller than $500 \mathrm{~nm}$ considering of monotonic increasing relationship between depth and etching time. Thus, $d / \lambda$ in short-wavelength range is larger than in long-wavelength range and the former in the reflectance spectra decreased faster than the latter. The depth of the texture etched for 10 minutes is 
Nano-Micro Lett. 3 (2), 129-134 (2011)/ http://dx.doi.org/10.3786/nml .v3i2.p129-134

about $500 \mathrm{~nm}$ as shown in Fig. 2(b), so the reflectance in long-wavelength range rapidly decreased due to the rise of $d / \lambda$. The possible physical reason for different reflectance trends of index-graded textures in different range is that shallow texture only scatters shortwavelength light effectively and deep texture simultaneously scatters both short- and long-wavelength light.

In spite of good antireflection properties, the Si NWs arrays by Ag-catalyzed etching are extremely rough and this must facilitate surface recombination. Therefore, we measured the effective lifetime $\left(\tau_{\text {eff }}\right)$ of the samples by means of MW-PCD technique to investigate influence of etching on surface recombination and the results are displayed in Tab. 1. According to MWPCD principles [18], $\tau_{\text {eff }}$ is related to bulk lifetime $\left(\tau_{b}\right)$ and surface recombination velocity $\left(\mathrm{S}_{R}\right)$ of the samples. Ag-catalyzed etching processes only change surface conditions and have no effect on $\tau_{b}$, so $\tau_{\text {eff }}$ solely depends on $\mathrm{S}_{R}$. As seen from Tab. 1, $\tau_{\text {eff }}$ decreases with the increasing of etching time and this means that $S_{R}$ increases with the increasing of the depths of textures. The deterioration of surface recombination is attributed to two possible reasons: enormous dangling bonds that continuously increased during etching and residual silver that is a deep-level impurity. They create many recombination centers in the forbidden band near the surface and cause serious surface recombination.

Table 1 The minority carrier lifetime of the samples etched by $\mathrm{HF}$ and $\mathrm{H}_{2} \mathrm{O}_{2}$ for different times.

\begin{tabular}{lcccc}
\hline Etching time $(\min )$ & 0 & 5 & 10 & 60 \\
\hline$\tau_{\text {eff }}(\mu \mathrm{s})$ & 1.577 & 1.538 & 1.471 & 1.259 \\
\hline
\end{tabular}

Obviously, serious surface recombination of Si NWs arrays will compensate for optimal antireflection if $\mathrm{Si}$ NWs are applied to replace pyramids and used as textures on conventional C-Si solar cells. Therefore, the key to reach high efficiency for Si NWs-textured solar cells will be compromised between surface antireflection and recombination. Furthermore, good passivation of Si NWs arrays textures is essential to reach high efficiency.

\section{Conclusion}

Si NWs arrays were produced by Ag-catalyzed etching techniques on single crystalline Si substrates. The Ag particle film plated on the Si substrates is made up of dendrites-like structures and its arrangement causes the appearance of the pits and nanowires. The ultralow reflectance below $3 \%$ and broadband antireflection effect from 400 to $1000 \mathrm{~nm}$ are realized. The excellent antireflection properties are attributed to index-graded profiles of the Si NWs arrays on subwavelength scales and suppression of reflection for different wavelength range depends on the ratio of textures depths and incident wavelength. However, surface recombination of Si NWs arrays deteriorates due to numerous dangling bonds and residual Ag particles.

\section{Acknowledgements}

This work was partly supported by Natural Science Foundation of China (No. 60876045), Shanghai Leading Basic Research Project (No. 09JC1405900), Shanghai Leading Academic Discipline Project (No. S30105), R\&D Foundation of SHU-SOENs PV Joint Lab (No. SS-E0700601). Part measurement was supported by Analysis and Testing Center of Shanghai University.

\section{References}

[1] A. Parretta, A. Sarnao, P. Tortora, H. Yakubu, P. Maddalena, J. Zhao and A. Wang, Opt. Commun. 172, 139 (1999). http://dx.doi.org/10.1016/ S0030-4018 (99)00561-1

[2] A. G. Aberle, Pro. Photovolt: Res. Appl. 8, 473 (2000). http://dx.doi.org/10.1002/1099-159X(200009/ 10) $8: 5 \$<\$ 473:$ :AID-PIP337 $\$>\$ 3.0 . \mathrm{CO} ; 2-\mathrm{D}$

[3] M. D. Kelzenberg, S. W. Boettcher, J. A. Petykiewicz, D. B. Turner-Evans, M. C. Putnam, E. L. Warren, J. M. Spurgeon, R. M. Briggs, N. S. Lewis and H. A. Atwater, Nature Mat. 9, 239 (2010). http://dx.doi. org/10.1038/nmat2727

[4] B. M. Kayes and H. A. Atwater, J. Appl. Phys. 97, 114302 (2005). http://dx.doi.org/10.1063/1. 1901835

[5] S. Chhajed, M. F. Schubert, J. K. Kim and E. F. Schubert, Appl. Phys. Lett. 93, 251108 (2008). http://dx. doi.org/10.1063/1.3050463

[6] P. B. Clapham and M.C. Hutley, Nature 244, 281 (1973). http://dx.doi.org/10.1038/244281a0

[7] H. Sai, H. Fujii, K. Arafune, Y. Ohshita and M. Yamaguchi, Appl. Phys. Lett. 88, 201116 (2006). http:// dx.doi.org/10.1063/1.2205173

[8] D. H. Raguin and G. M. Morris, Appl. Opt. 32, 1154 (1993). http://dx.doi.org/10.1364/A0.32.001154

[9] C. Wu, C. H. Crouch, L. Zhao, J. E. Carey, R. Younkin, J. A. Levinson, E. Mazur, R. M. Farrell, P. Gothoskar and A. Karger, Appl. Phys. Lett. 78, 1850 (2001). http://dx.doi.org/10.1063/1.1358846

[10] K. Q. Peng, Y. J. Yan, S. P. Gao and J. Zhu, Adv. Mater. 14, 1164 (2002). http://dx.doi.org/ 10.1002/1521-4095(20020816) 14: 16\$<\$1164: : AID-ADMA1164\$>\$3. 0. CO; 2-E

[11] C. Yang, C. J. Barrelet, F. Capasso and C. M. Lieber, Nano Lett. 6, 2929 (2006). http://dx.doi.org/10. $1021 / \mathrm{nl} 062314 \mathrm{~b}$

[12] L. T. Canham: Appl. Phys. Lett. 57, 1046 (1990). http://dx.doi.org/10.1063/1.103561

[13] M. L. Zhang, K. Q. Peng, X. Fan, J. S. Jie, R. Q. Zhang, S. T. Lee and N. B. Wong, J. Phys. 
Chem. C 112, 4444 (2008). http://dx.doi.org/10. 1021/jp077053o

[14] Y. H. Ogata, K. Kobayashi and M. Motoyama, Curr. Opin. Solid State Mater. Sci. 10, 163 (2006). http:// dx.doi.org/10.1016/j.cossms. 2007.02.001

[15] K. Q. Peng, J. J. Hu, Y. J. Yan, Y. Wu, H. Fang, Y. Xu, S. T. Lee and J. Zhu, Adv. Funct. Mater. 16, 387 (2006). http://dx.doi.org/10.1002/adfm. 200500392
[16] J. C. Maxwell Garnett, Philos. Trans. R. Soc. London 205, 237 (1906). http://dx.doi.org/10.1098/rsta. 1906.0007

[17] R. B. Stephens and G. D. Cody, Thin Solid Films 45, 19 (1977). http://dx.doi.org/10.1016/ 0040-6090 (77) 90197-3

[18] D. K. Schroder: Semiconductor Material and Device Characterization (John Wiley \& Sons., Publication, New Jersey 2006). 